\title{
Reviewer Acknowledgment
}

Decision depends on the goodwill of many experts who help review manuscripts that have been submitted for publication. In addition to the Consulting Editors, the following individuals reviewed manuscripts that were considered for the 2021 volume. The Editor acknowledges all reviewers and thanks them for their time and effort.

\begin{tabular}{|c|c|c|}
\hline Woo-Young Ahn & Russell Golman & Bradley Morris \\
\hline Poonam Arora & Richard Gonzalez & Johannes Muller-Trede \\
\hline Pavel Atanasov & Yael Grushka-Cockayne & Ryan O. Murphy \\
\hline Yigal Attali & David Hagmann & Thomas Mussweiler \\
\hline Winton $\mathrm{Au}$ & Nir Halevy & Gideon Nave \\
\hline & Shlomo Hareli & Nathan Novemsky \\
\hline Aurelien Baillon & Laura Hatz ${ }^{*}$ & Henrik Olsson \\
\hline Saurabh Bansal & Claire Heard & Ayse Onculer \\
\hline Maya Bar-Hillel & Stefan Herzog & Danny Oppenheimer \\
\hline Daniel Benjamin & Mark Himmelstein & Andreas Ortmann \\
\hline Daniel Bennett & Emily Ho & 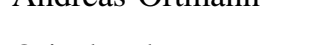 \\
\hline Gary Bolton & Guy Hochman & Ori Plonsky \\
\hline Bryan Bonner & Shu Huang* & Drazen Prelec \\
\hline Jerome R. Busemeyer & Joel Huber & Tim Rakow \\
\hline & Eric Johnson & Rob Ranyard \\
\hline Arthur Carvalho & Yaakov Kareev & Michael Razen \\
\hline Daniel R. Cavagnaro & Elena Katok & Barry Rosenfeld \\
\hline Nick Chater & Steffen Keck & Yuval Rottenstriech \\
\hline Zhi Chen & Martin Kocher & Jay Russo \\
\hline Jiuqing Cheng & Gregory Koop & \\
\hline Shoham Choshen-Hillel & Goedele Krekels & Ville Satopaa \\
\hline Fintan Costello & Kristine Kuhn & Thomas Schlösser \\
\hline Gregory Edward Cox & Ralf Kurvers & $\begin{array}{l}\text { Sandra Schneider } \\
\text { Andrew Schotter }\end{array}$ \\
\hline Junyi Dai & Richard Larrick & Thomas Schultze \\
\hline Jason Dana & Tomas Lejarraga & Matthias Seifert \\
\hline Peter Dayan & Peter Lichtenberg & Rajiv Sethi \\
\hline Enrico Diecidue & Shenghua Luan & Sahana Shankar* \\
\hline Ning Du & Ashley Luckman & Rashmi Sharma \\
\hline Nicholas Epley & Christian Luhmann & Leonidas Spiliopoulos \\
\hline Eyl Ert & Boris Maciejovsky & Mark Steyvers \\
\hline Ilan Fischer & Rui Mata & $\begin{array}{l}\text { Giles Story } \\
\text { James Sundali }\end{array}$ \\
\hline Shane Frederick & $\begin{array}{l}\text { John McCoy } \\
\text { Blake McShane }\end{array}$ & Kinne $>_{2}$ \\
\hline Celia Gaertig & Barbara Mellers & Robert Scott Tindale \\
\hline Colleen Giblin & Jason Merrick & Kevin Trutmann* \\
\hline
\end{tabular}




$\begin{array}{lll}\text { Fabio Tufano } & \text { Doug Wedell } & \text { Marc Wyszynski } \\ \text { Canan Ulu } & \text { Andreas Wilke } & \text { Ilan Yaniv } \\ \text { Tom Wilkening } & \text { Eldad Yechiam } \\ \text { Paul van Bruggen } & \text { Paul Windschitl } & \text { Gal Zauberman } \\ \text { Lyn van Swol } & \text { Jens Witkowski } & \text { Ting Zhang } \\ \text { Winjand van Tilburg } & \text { Yin Wu } & \text { Rami Zwick } \\ \text { Elke Weber } & \text { Dirk Wulff } & \\ & & \\ * \text { * Denotes a reviewer who co-reviewed under the supervision of a primary reviewer. }\end{array}$

